\title{
Reproducibility Between Three-Dimensional Turbo Spin-Echo and Two-Dimensional Dual Inversion Recovery Turbo Spin-Echo for Coronary Vessel Wall Imaging in Kawasaki Disease
}

Koji Matsumoto ( $\nabla$ matumoto@chiba-u.jp )

Department of Radiology, Chiba University Hospital

Hajime Yokota

Diagnostic Radiology and Radiation Oncology, Graduate School of Medicine, Chiba University

Takafumi Yoda

Department of Radiology, Chiba University Hospital

\section{Ryota Ebata}

Department of Pediatrics, Graduate School of Medicine, Chiba University

Hiroki Mukai

Department of Radiology, Chiba University Hospital

\section{Yoshitada Masuda}

Department of Radiology, Chiba University Hospital

\section{Takashi Uno}

Diagnostic Radiology and Radiation Oncology, Graduate School of Medicine, Chiba University

\section{Research Article}

Keywords: Kawasaki disease, coronary artery, vessel wall imaging, black blood imaging, turbo spin-echo

Posted Date: October 28th, 2021

DOI: https://doi.org/10.21203/rs.3.rs-980453/v1

License: (c) (1) This work is licensed under a Creative Commons Attribution 4.0 International License. Read Full License 


\section{Abstract}

Background: Magnetic resonance vessel wall imaging is desirable for evaluating Kawasaki disease (KD)associated coronary arterial lesions.

Purpose: To evaluate reproducibility of three-dimensional turbo spin-echo (3D-TSE) and two-dimensional dual inversion-recovery turbo spin-echo (2D-DIR-TSE) for coronary vessel wall imaging in KD.

Methods: Ten patients were prospectively enrolled. Coronary vessel wall imaging with axial-slice orientation 3D-TSE and 2D-DIR-TSE were acquired for cross-sectional images in aneurysmal and normal regions. Lumen area (LA), wall area (WA), and normalized wall index (NWI) of cross-sectional images were measured in both regions. Reproducibility between 3D-TSE and 2D-DIR-TSE was evaluated via intraclass correlation coefficients (ICCs) and Bland-Altman plots.

Results: 48 points (aneurysmal, 27; normal, 21) were evaluated. There were high ICCs between 3D-TSE and 2D-DIR-TSE in LA (0.95) and WA (0.95). In aneurysmal regions, 95\% limits of agreement were LA, WA, and NWI of -29.9-30.4 mm², $-18.8-15.0 \mathrm{~mm}^{2}$, and $-0.22-0.20$, respectively. In normal regions, the $95 \%$ limits of agreement were LA, WA, and NWI of $-4.44-4.38 \mathrm{~mm}^{2},-3.51-4.30 \mathrm{~mm}^{2}$, and $-0.14-0.16$, respectively. No fixed and proportional biases between 3D-TSE and 2D-DIR-TSE images in aneurysmal and normal regions were noted.

Conclusions: 3D-TSE was reproducible with conventional 2D-DIR-TSE for coronary vessel wall assessment on $\mathrm{KD}$.

\section{Introduction}

Kawasaki disease (KD) is an acute febrile illness of unknown etiology that usually occurs in children below five years of age and is a systemic vasculitis syndrome [1]. Coronary arteritis is caused in the majority of patients with acute KD [2], often resulting in coronary artery aneurysms. Aneurysm formation in $\mathrm{KD}$ is considered secondary to the destruction of the internal elastic membrane because of severe coronary angiitis [3]. Coronary artery aneurysms can rupture, thrombose, and lead to the development of stenotic lesions. Its serious coronary complications reportedly cause ischemic heart disease in children and are now the most common cause of pediatric coronary disease worldwide [4].

Coronary magnetic resonance angiography (MRA) using three-dimensional (3D) balanced steady-state free precession imaging, a minimally invasive procedure, is widely used for evaluating coronary arterial lesions in KD [5]. However, MRA does not allow the sufficient evaluation of coronary artery wall changes, such as thrombi and intimal proliferation. Intravascular ultrasound (IVUS) or optical coherence tomography (OCT) is the standard approach for coronary artery wall assessments [6, 7]. However, IVUS and OCT are invasive techniques with non-negligible risks and are difficult to perform routinely.

Transthoracic echocardiography (TTE) [8] can be generally used to evaluate the coronary vessel wall up to early childhood. Nonetheless, it is difficult to conduct TTE after adolescence. This necessitates a non- 
invasive method that can be examined frequently from the acute to the follow-up phase. Magnetic resonance vessel wall imaging is desirable for evaluating KD-associated coronary arterial lesions [9].

Previous reports have used two-dimensional dual inversion-recovery turbo spin-echo (2D-DIR-TSE) $[9,10]$ and three-dimensional turbo spin-echo (3D-TSE) [11] imaging for pediatric coronary vessel wall imaging. 2D-DIR-TSE imaging is often challenging to set the field-of-view (FOV) in clinical cases. This can be attributed to the occurrence of coronary artery aneurysms in multiples and at bifurcations. This necessitates 3D-TSE imaging that facilitates extensive coverage. Any section or direction can be reconstructed and visualized using wide-FOV-imaging-derived 3D volume data. In contrast, 2D-DIR-TSE imaging has the advantage of obtaining a cross-sectional image with high in-plane resolution in a short scan time. The disadvantages of 3D-TSE imaging include motion artifacts because of the long scan time. In addition, the flow direction, with respect to the slice encoding direction, affects the suppression of the blood signal. The problems of 2D-DIR-TSE imaging have not been completely evaluated previously. Thus, it is necessary to compare the reproducibility and effectiveness of 3D-TSE and 2D-DIR-TSE imaging. Therefore, we aimed to perform qualitative and quantitative evaluations of cross-sectional vessel wall images collected by 3D-TSE and 2D-DIR-TSE imaging in KD, and to compare their reproducibility.

\section{Methods}

\section{Subjects}

Approval was provided by Chiba University Hospital (approved number, 3901; Registered 14 December 2020) and written informed consent was obtained from all patients or their family. This study was performed in accordance with relevant guidelines and regulations, including the Declaration of Helsinki. We prospectively enrolled ten patients (five each female and male; mean age \pm standard deviation [SD], $12.9 \pm 7.2$ years; range, $5-25$ years) who underwent cardiac magnetic resonance imaging (MRI) between September 2015 and October 2019. These patients were undergoing follow-up care for a transient dilated lesion of the coronary artery during the acute phase of KD or coronary arterial lesions, such as aneurysms and stenosis resulting from $\mathrm{KD}$.

TTE and X-ray coronary angiography (XCA) revealed aneurysms at 15 sites in 10 patients (right coronary artery [RCA], five; left coronary artery [LCA], 10). No thromboses were identified by TTE or XCA. The heart rates during MRI ranged between 63-81 beats per minute (BPM) (mean \pm SD: $72.1 \pm 6.6 \mathrm{BPM}$ ). Of the ten patients, five aged $<9$ years underwent MRI examination under sedation. To achieve mild patient sedation during the MRI examination, they were administered sodium trichloroethylene phosphate syrup ( 0.7 to 0.8 $\mathrm{mL} / \mathrm{kg})$. However, they were administered thiopental sodium $(2-3 \mathrm{mg} / \mathrm{kg})$ or midazolam $(0.2 \mathrm{mg} / \mathrm{kg})$ via IV infusion if the syrup was ineffective, to achieve unconscious sedation for the duration of imaging.

\section{Data acquisition}

All MRI studies were performed on a 1.5-Tesla MRI unit (Intera Achieva Nova Dual Release 5, Philips Healthcare, Best, The Netherlands) using a chest-phased array coil. First, we used a high-resolution 2D 
cine scan with an axial slice orientation to identify the precise period of minimal cardiac motion in each patient [12]. Subsequently, we performed MRA using 3D balanced steady-state free precession (3D balanced turbo field-echo) with an axial slice orientation to confirm the target part and image the entire heart. Next, 3D-TSE imaging was performed with axial slice orientation in the proximal regions of the coronary artery. Moreover, we acquired cross-sectional images of the aneurysm from multiple crosssections (e.g., center, proximal, and distal portions) and images of normally appearing juxta-proximal and juxta-distal points of the aneurysms with 2D-DIR-TSE imaging.

3D-TSE images were obtained with the following parameters: (i) a free-breathing navigator-gated and cardiac-triggered 3D turbo spin-echo with a repetition time (TR) equivalent to the duration of one heartbeat (698 ms to $896 \mathrm{~ms}$ ); (ii) an echo time (TE) of $36 \mathrm{~ms}$ to $62 \mathrm{~ms}$; (iii) a refocusing flip angle of 50 ; (iv) a turbo factor of 14 to 22 ; (v) an acquired spatial resolution of $1.17 \times 1.25 \times 1.40 \mathrm{~mm}$ (reconstruction, $0.55 \times 0.55 \times 0.70 \mathrm{~mm}$ ); (vi) a slice number of 60; (vii) spectral inversion recovery (SPIR); and (viii) flowsensitizing to suppress the blood signal [13]. The acquisition window was set to $40-70 \mathrm{~ms}$, depending on the period of minimum cardiac motion for each patient. The total scan time was 3 min $53 \mathrm{~s}$, with 100\% navigator efficiency (calculated for a heart rate of $70 \mathrm{BPM}$ ). We used a $50^{\circ}$ refocusing flip angle to increase the saturation. Flowing spin in fast spin-echo imaging arises from two primary mechanisms as follows: intravoxel dephasing from isochromats with different velocities and mixing among pathways involving different combinations of spin echoes and stimulated echoes [14]. The use of low refocusing flip angles further promotes the phase dispersion of flowing spins [14]. Furthermore, it slows the decay of the signal from stationary tissues, thereby allowing longer echo trains or a narrower echo spacing [15]. In addition, we applied an SPIR pulse to suppress the epicardial fat signals.

In contrast, 2D-DIR-TSE images were obtained with the following parameters: (i) a free-breathing navigator-gated and cardiac-triggered 2D turbo spin-echo with a TR equivalent to the duration of one heartbeat (698 ms to $896 \mathrm{~ms}$ ); (ii) a TE of $20 \mathrm{~ms}$ [10]; (iii) a turbo factor of 9; (iv) a resolution of $0.98 \times 0.96 \times 4.00 \mathrm{~mm}$ (acquired spatial reconstruction, $0.49 \times 0.49 \times 4.00 \mathrm{~mm}$ ); and $(\mathrm{v})$ an SPIR and acquisition window of $45 \mathrm{~ms}$. The total scan time was $26 \mathrm{~s}$, with $100 \%$ navigator efficiency (calculated for a heart rate of $70 \mathrm{BPM})$.

All sequences were equipped with a right hemi-diaphragmatic prospective real-time navigator for suppressing the respiratory motion artifact in patients with free breathing [16]. Data were accepted if the lung-liver interface was within the end-expiratory gating window (1-4 mm). However, they were rejected and reacquired during the subsequent inter-beat interval.

\section{Postprocessing}

Coronary vessel walls were evaluated separately in both the aneurysmal and normal regions diagnosed via TTE or XCA. A coronary aneurysm was diagnosed if the internal lumen diameter was $>4.0 \mathrm{~mm}$ or on observing a distension of a part of a coronary vessel of up to one and a half times the diameter of an adjacent normal segment [17]. 
To evaluate the coronary vessel wall and lumen size, we reformatted the 3D-TSE dataset for same-angle cross-sectional images as 2D-DIR-TSE on a commercial workstation (OsiriX Dicom viewer Version 12.0, Pixmeo SARL, Switzerland). To minimize digitization errors, the cross-sectional images were reconstructed using 768 matrices. In the coronary vessel wall images, we set the window level and width at the level to diminish the intraventricular blood signal.

\section{Visual grading}

Two board-certified radiologists with 15 and 13 years of experience, independently evaluated all coronary vessel wall images. The quality of the lumen boundary and outer wall boundary of each cross-sectional image was visually graded using a four-point grade (4, excellent; 3 , good; 2 , fair; and 1, bad). Table 1 summarizes the criteria for the four-point grade [18]. Any discrepancies between the two readers were resolved by consensus.

Table 1

Image quality assessment

\begin{tabular}{|lll|}
\hline Score & Grading & Description \\
\hline \multicolumn{2}{|l|}{ Lumen boundary visualization quality } \\
\hline 2 & Bad & Strong flow artifacts, lumen boundary was not visible, not diagnostic. \\
\hline 3 & Fair & Moderate flow artifacts, a part of lumen boundary was unclear. \\
\hline 4 & Excellent & No flow artifacts, lumen boundary was clearly shown. \\
\hline Outer wall boundary visualization quality \\
\hline 1 & Bad & More than $1 / 3$ of the vessel wall was not visualized. \\
\hline 2 & Fair & Vessel wall was unclear, but $2 / 3$ circumference was visible. \\
\hline 3 & Good & Vessel wall was visible, but a portion was unclear. \\
\hline 4 & Excellent & Entire vessel wall was clearly visible. \\
\hline
\end{tabular}

\section{Measuring the lumen area, wall area, and normalized wall index}

The lumen and outer wall contours of each cross-sectional image were manually traced using the OsiriX Dicom viewer (https://www.osirix-viewer.com/). Based on these contours, the software program automatically calculated the lumen area (LA) and total vessel area (TVA) for each analyzed image. The wall area (WA) was measured by subtracting the LA from the TVA. In addition, to adjust for differences in the vessel size within each patient, we calculated a normalized wall index (NWI) [19]. The NWI was calculated as follows: 


\section{NWI=WA/TVA}

Figure 1. Regions of interest segmentation. The coronary magnetic resonance angiography-collapsed partial maximum intensity projection image demonstrates right coronary artery (RCA) segments 1 in a 19year-old boy with Kawasaki disease (a). At 6 months of age, he experienced a coronary artery aneurysm of up to $5.9 \mathrm{~mm}$ in RCA segments 1 . It is currently involuted and divided into normal regions. The white line depicts the slice position in the two-dimensional dual inversion-recovery turbo spin-echo crosssectional image $b$. Image $c$ is an enlarged image of the measurement location of $b$. The lumen of the coronary artery (red dotted line) and the boundaries of the wall (yellow solid line) have been manually contoured (c). Subsequently, the lumen area (LA), the total vessel area (TVA) and the wall area (WA) have been measured (d, e, f) (Ao, aorta; RA, right atrium; and RV, right ventricle).

\section{Inter- and intra-rater reproducibility}

To determine the intra-rater reproducibility, an experienced rater (A, 20 years of image analysis experience) analyzed all cross-sectional images. The recall bias was reduced to a minimum by using a time frame of approximately 180 days between the first and second image analyses. In contrast, to determine the inter-rater reproducibility, another rater (B, 4 years of image analysis experience) independently analyzed similar images as A (Fig. 2) [19]. Both raters were blinded to the patient's characteristics and results. The blinding was ensured by replacing each patient's image identifier with a pre-assigned unique study number.

\section{Statistical analyses}

All statistical analyses were performed using R version 3.6.3 (The R Foundation for Statistical Computing, Vienna, Austria). To compare the visual grading of 3D-TSE and 2D-DIR-TSE images, we performed Wilcoxon signed rank tests. $P$-values $<0.05$ were considered statistically significant. To compare the reproducibility between 3D-TSE and 2D-DIR-TSE images, we used intraclass correlation coefficients (ICCs) and Bland-Altman plots. Fixed and proportional biases were defined as significant with $P$-values $<0.05$, using a paired t-test and linear regression.

ICCs were used to evaluate the inter-rater and intra-rater reproducibility.

\section{Results}

We evaluated a total of 48 coronary artery points (aneurysmal regions, 27; normal regions, 21). The actual scan time for each case was approximately 9-11 min and 1-2 min for 3D-TSE imaging and 2DDIR-TSE imaging, respectively. All imaging was performed during mid-diastole, a quiescent period in the cardiac cycle. Table 2 summarizes the American Heart Association segments and the number of points evaluated from all patients with KD.

Table 2. The number of points evaluated in 10 patients with Kawasaki disease 


\begin{tabular}{llll} 
Patients (age [yr] \& gender) & AHA segment(s) & Aneurysm & Normal \\
\hline Case 1 (8F) & 6 & 4 & 1 \\
\hline Case 2 (8M) & 5 & N/A & 3 \\
\hline Case 3 (6F) & 6 & N/A & 2 \\
\hline Case 4 (16F) & 1 & 3 & 2 \\
\hline Case 5 (25M) & 5 & 5 & N/A \\
\hline Case 6 (11F) & 1 & N/A & 3 \\
\hline Case 7 (6M) & 5 & N/A & 3 \\
\hline Case 8 (19M) & 6 & 3 & 1 \\
& 1 & N/A & 1 \\
\hline Case 9 (5M) & 2 & 3 & N/A \\
\hline Case 10 (20F) & 1 & N/A & 3 \\
\hline Total & 5 & N/A & 1 \\
\hline & 6 & N/A & 1 \\
\hline & 2 & 3 & N/A \\
\hline & 6 & 3 & N/A \\
\hline & 5 & 3 & N/A \\
\hline
\end{tabular}

AHA, American Heart Association; N/A, not applicable.

Table 3 summarizes the visual grading of the 3D-TSE and 2D-DIR-TSE images. Grade 3 or higher points in the aneurysmal regions were $25.9 \%$ and $18.5 \%$ at the lumen boundary, and $22.2 \%$ and $18.5 \%$ at the outer wall boundary for 3D-TSE and 2D-DIR-TSE images, respectively. Conversely, grade 3 or higher points in the normal regions were $95.2 \%$ for both 3D-TSE and 2D-DIR-TSE images at the lumen boundary, and $42.9 \%$ and $52.4 \%$ for 3D-TSE and 2D-DIR-TSE images, respectively, at the outer wall boundary. There were no significant differences between the 3D-TSE and 2D-DIR-TSE images in the lumen boundary and outer wall boundary. At the lumen boundary in the aneurysm regions, the 3D-TSE images had a higher visual grade than the 2D-DIR-TSE images. However, this difference was statistically insignificant $(P=0.073)$. At the outer wall boundary in the normal regions, the 2D-DIR-TSE images had a higher visual grade than the 3D-TSE images. Nonetheless, this difference was statistically insignificant $(P=0.065)$. Coronary artery points with a visual grade of 1 in either 3D-TSE or 2D-DIR-TSE images were removed from subsequent analyses. 
Table 3. The summary of visual grading: Aneurysmal region, $n=27$; Normal region, $n=21$

\begin{tabular}{llllllllll} 
& \multicolumn{3}{c}{ Lumen boundary } & \multicolumn{5}{c}{ Outer wall boundary } \\
\hline & Grade & \multicolumn{2}{l}{ Aneurysm } & \multicolumn{2}{l}{ Normal } & \multicolumn{2}{l}{ Aneurysm } & \multicolumn{2}{l}{ Normal } \\
\hline 3D-TSE & 4 & 1 & $(3.7 \%)$ & 13 & $(61.9 \%)$ & 3 & $(11.1 \%)$ & 0 & $(0 \%)$ \\
\hline & 3 & 6 & $(22.2 \%)$ & 7 & $(33.3 \%)$ & 3 & $(11.1 \%)$ & 9 & $(42.9 \%)$ \\
\hline & 2 & 15 & $(55.6 \%)$ & 1 & $(4.8 \%)$ & 9 & $(33.3 \%)$ & 10 & $(47.6 \%)$ \\
\hline 2D-DIR-TSE & 1 & 5 & $(18.5 \%)$ & 0 & $(0 \%)$ & 12 & $(44.4 \%)$ & 2 & $(9.5 \%)$ \\
\hline & 3 & 0 & $(0 \%)$ & 13 & $(61.9 \%)$ & 3 & $(11.1 \%)$ & 5 & $(23.8 \%)$ \\
\hline & 2 & 15 & $(55.6 \%)$ & 1 & $(4.8 \%)$ & 12 & $(44.4 \%)$ & 9 & $(42.9 \%)$ \\
\hline 3D vs. 2D & 1 & 7 & $(25.9 \%)$ & 0 & $(0 \%)$ & 10 & $(37.0 \%)$ & 1 & $(4.8 \%)$ \\
\hline
\end{tabular}

3D-TSE, three-dimensional turbo spin-echo; 2D-DIR-TSE, two-dimensional dual inversion-recovery turbo spin-echo.

Table 4 lists the LA, WA, and NWI. We observed high ICCs between 3D-TSE and 2D-DIR-TSE images in the LA and WA (LA, 0.95; WA, 0.95). There were no significant differences between the 3D-TSE and 2D-DIRTSE images in the aneurysmal and normal regions.

Table 4. A comparison of the area measurements between 3D-TSE and 2D-DIR-TSE: Aneurysmal region, $\mathrm{n}=15$; Normal region, $\mathrm{n}=19$

\begin{tabular}{|c|c|c|c|c|c|c|}
\hline & $\mathrm{LA}\left(\mathrm{mm}^{2}\right)$ & & WA $\left(\mathrm{mm}^{2}\right)$ & & NWI & \\
\hline & Aneurysm & Normal & Aneurysm & Normal & Aneurysm & Normal \\
\hline 3D-TSE & $50.6 \pm 35.1$ & $8.6 \pm 2.4$ & $36.1 \pm 15.6$ & $7.9 \pm 2.1$ & $0.45 \pm 0.10$ & $0.48 \pm 0.05$ \\
\hline 2D-DIR-TSE & $50.3 \pm 39.2$ & $8.7 \pm 2.7$ & $38.0 \pm 18.7$ & $7.5 \pm 1.7$ & $0.46 \pm 0.14$ & $0.47 \pm 0.07$ \\
\hline $3 D$ vs. $2 D$ & $P=0.948$ & $P=0.956$ & $P=0.407$ & $P=0.399$ & $P=0.736$ & $P=0.540$ \\
\hline
\end{tabular}

Table 5 outlines the inter-rater and intra-rater reproducibility results. The inter- and intra-rater ICCs of all area measurements were $>0.8$, thus indicating excellent reproducibility.

Table 5. The list of intraclass correlation coeffcients between area measurement on inter- and intra-rater reproducibility, $n=34$ 


\begin{tabular}{lllllll}
\multicolumn{1}{c}{ LA } & \multicolumn{5}{c}{ WA } & NWI \\
& 3D-TSE & 2D-DIR-TSE & 3D-TSE & 2D-DIR-TSE & 3D-TSE & 2D-DIR-TSE \\
\hline Inter- & $0.99[0.97$, & $1.00[0.99$, & $0.98[0.95$, & $0.98[0.97$, & $0.74[0.50$, & $0.80[0.57$, \\
rater & $0.99]$ & $1.00]$ & $0.99]$ & $0.99]$ & $0.87]$ & $0.91]$ \\
\hline Intra- & $0.99[0.97$, & $0.99[0.98$, & $0.95[0.90$, & $0.97[0.93$, & $0.73[0.52$, & $0.74[0.49$, \\
rater & $0.99]$ & $0.99]$ & $0.98]$ & $0.99]$ & $0.86]$ & $0.87]$ \\
\hline
\end{tabular}

[95\% confidence interval]

Fig. 3 depicts the Bland-Altman plots of the LA, WA, and NWI between the 3D-TSE and 2D-DIR-TSE images. The $95 \%$ limits of agreement were LA, WA, and NWI of -29.9 to $30.4 \mathrm{~mm}^{2},-18.8$ to $15.0 \mathrm{~mm}^{2}$, and -0.22 to 0.20 in the aneurysmal regions, and -4.44 to $4.38 \mathrm{~mm}^{2},-3.51$ to $4.30 \mathrm{~mm}^{2}$, and -0.14 to 0.16 in the normal regions, respectively. There were no fixed and proportional biases between the 3D-TSE and 2D-DIR-TSE images in the aneurysmal and normal regions (Table 4 and Fig. 3). Fig. 4 depicts the representative 3D-TSE and 2D-DIR-TSE images.

\section{Discussion}

We investigated the reproducibility of 3D-TSE and conventional 2D-DIR-TSE imaging in coronary vessel wall imaging in KD. To our knowledge, this is the first study to compare the 3D-TSE and 2D-DIR-TSE images in the coronary arteries. Qualitative and quantitative evaluations suggested reproducibility between the 3D-TSE and 2D-DIR-TSE images.

Despite slower flow velocity and larger influence of turbulence in the aneurysmal region than those in the normal region, the 3D-TSE images were equal to or better than the 2D-DIR-TSE images in the visual grade of the lumen boundary. High-resolution, 3D-TSE imaging with variable flip angle trains (e.g., VISTA, SPACE, and CUBE) is advantageous for vessel wall imaging at various sites, considering its high scan efficiency, enabled by the use of long echo trains and its intrinsic black blood effect [15, 20-23]. 3D-TSE imaging reportedly exerts a strong suppressive effect on blood flow signals in the coronary arteries. We used 3D-TSE imaging to select axial slice orientation. We observed suppression in coronary artery vessel wall imaging when the flow direction was close to parallel to the slice encoding direction. However, there was no suppression when the direction was close to perpendicular [11]. Aneurysms are often located in the proximal portion of the coronary artery [24]. By axially collecting the proximal portion of the coronary artery, the blood flow direction and the slice encoding direction become close to parallel, such that the blood flow signal was effectively suppressed.

In normal regions, the 3D-TSE images were equal to or less than the 2D-DIR-TSE images in the visual grade of the outer wall boundary. 3D-TSE imaging suggestively has a longer shot duration and scan time than 2D-DIR-TSE imaging, which increases the effects of heart action and respiration movement. In follow-up examination of KD, it is necessary not only to detect high-intensity thromboses but also to depict detailed anatomical structures, such as aneurysm regression. Considering the small anatomical 
size of coronary arteries and the movement during heart action and respiration, coronary vessel wall imaging remains more challenging than imaging of other vascular beds [25]. In a previous study, autopsy studies in subjects who did not die of coronary artery disease have revealed that the coronary vessel wall is typically $0.4-0.8 \mathrm{~mm}$ thick [26]. A previous report on the impact of spatial resolution on the accuracy of vessel WA measurement in simulations and phantom studies mentioned that a resolution $\leq 4$ pixels across the wall leads to an overestimation of over $20 \%$ [27]. Moreover, coronary wall imaging reportedly requires a spatial resolution of $0.5 \mathrm{~mm}$ to $1 \mathrm{~mm}[28,29]$. In the present study, the acquired spatial resolution of $1.17 \times 1.25 \times 1.40 \mathrm{~mm}$ was further reconstructed to $0.55 \times 0.55 \times 0.70 \mathrm{~mm}$. Nonetheless, it is necessary to further improve the spatial resolution with technological advancements in future.

3D-TSE and 2D-DIR-TSE images had high ICCs and no bias, suggesting the images were reproducible. A previous study investigated the reproducibility of the internal diameter between 3D-TSE images and MRA [11]. Larger the diameter of the aneurysm, narrower was the internal diameter of the 3D-TSE image than that of the MRA image. This may be attributed to laminar flow proximal to the vessel wall and increased turbulent flow, caused by changes in the shape of the vessel lumen. In contrast, there was no bias in the internal diameter between 3D-TSE images and MRA in normal regions, thereby generating the expected range. The signal intensity of conventional 2D-DIR-TSE imaging relies on the inflow of fresh blood into the imaging slice between selective re-inversion and imaging. Therefore, aneurysmal regions are susceptible to flow direction and velocity. 2D-DIR-TSE images have a strong directivity in the acquisition section and flow direction, similar to 3D-TSE images.

The inter- and intra-rater reproducibility in the area measurement suggested that 3D-TSE images may be suitable for follow-up examination of the coronary vessel wall. 3D-TSE imaging also allows for an easy visualization of accurate cross-sections, with less partial volume effects following post-processing.

While this study generated significant findings, there were several limitations. First, we did not compare MR coronary vessel wall imaging with IVUS or OCT [11,30], a standard approach for coronary artery wall assessments. However, IVUS is an invasive technique with non-negligible risks and is difficult to perform routinely. Second, the voxel sizes of 3D-TSE and 2D-DIR-TSE imaging did not match. However, there occurs a trade-off between high-resolution imaging and increased scan time, which can increase patient discomfort.

In conclusion, 3D-TSE imaging was reproducible with conventional 2D-DIR-TSE imaging for coronary vessel wall assessment of KD. The aneurysmal regions necessitate careful WA assessment, owing to the residual lumen signals of 3D-TSE and 2D-DIR-TSE images. 3D-TSE imaging may be suitable for follow-up examinations of KD because of its wide FOV, accurate cross-sectional images, and the reproducibility of area measurements.

\section{Declarations}

Acknowledgements 
The authors would like to thank, application staff, Atsushi Takemura at Philips Healthcare for providing information about 3D-TSE imaging.

\section{Author contributions}

$\mathrm{KM}$ and $\mathrm{HY}$ were major contributors in study design, interpretation of data and writing and revising the manuscript. KM and TY have acquired the data. KM, HY, TY and HM performed data analysis. RE, YM and TU collected clinical information. All authors read and approved the final manuscript.

\section{Additional Information}

The authors declare that they have no competing interests.

\section{References}

1. Newburger, J. W., et al. Diagnosis, treatment, and long-term management of Kawasaki disease: a statement for health professionals from the Committee on Rheumatic Fever, Endocarditis, and Kawasaki Disease, Council on Cardiovascular Disease in the Young, American Heart Association. Pediatrics 114, 1708-1733 (2004).

2. Takahashi, K., Oharaseki, T. \& Naoe, S. Pathological study of postcoronary arteritis in adolescents and young adults: with reference to the relationship between sequelae of Kawasaki disease and atherosclerosis. Pediatr. Cardiol. 22, 138-142 (2001).

3. Onouchi, Z., Tomizawa, N., Goto, M., Nakata, K. \& Fukuda, M. Cardiac involvement and prognosis in acute mucocutaneous lymph node syndrome. Chest 68, 297-301 (1975).

4. Kitamura, S. The role of coronary bypass operation on children with Kawasaki disease. Coron. Artery Dis. 13, 437-447 (2002).

5. Takemura, A., et al. Utility of coronary MR angiography in children with Kawasaki disease. AJR Am. J. Roentgenol. 188, W534-W539 (2007).

6. Mintz, G. S. Clinical utility of intravascular imaging and physiology in coronary artery disease. J. Am. Coll. Cardiol. 64, 207-222 (2014).

7. Kume, T., et al. Measurement of the thickness of the fibrous cap by optical coherence tomography. Am. Heart J. 152, 755.e1-755.e4 (2006).

8. Capannari, T. E., Daniels, S. R., Meyer, R. A., Schwartz, D. C. \& Kaplan, S. Sensitivity, specificity and predictive value of two-dimensional echocardiography in detecting coronary artery aneurysms in patients with Kawasaki disease. J. Am. Coll. Cardiol. 7, 355-360 (1986).

9. Suzuki, A. et al. Magnetic resonance coronary angiography to evaluate coronary arterial lesions in patients with Kawasaki disease. Cardiol. Young 16, 563-571 (2006).

10. Greil, G. F., et al. Coronary magnetic resonance angiography and vessel wall imaging in children with Kawasaki disease. Pediatr. Radiol. 37, 666-673 (2007). 
11. Matsumoto, K., et al. Coronary vessel wall visualization via three-dimensional turbo spin-echo black blood imaging in Kawasaki disease. Magn. Reson. Imaging 62, 159-166 (2019).

12. Kim, W. Y. et al. Impact of bulk cardiac motion on right coronary MR angiography and vessel wall imaging. J. Magn. Reson. Imaging 14, 383-390 (2001).

13. Hinks, R. S. \& Constable, R. T. Gradient moment nulling in fast spin echo. Magn. Reson. Med. 32, 698-706 (1994).

14. Storey, P., et al. Tailoring the flow sensitivity of fast spin-echo sequences for noncontrast peripheral MR angiography. Magn. Reson. Med. 64, 1098-1108 (2010).

15. Busse, R. F., et al. Effects of refocusing flip angle modulation and view ordering in 3D fast spin echo. Magn. Reson. Med. 60, 640-649 (2008).

16. Stuber, M., Botnar, R. M., Danias, P. G., Kissinger, K. V. \& Manning, W. J. Submillimeter threedimensional coronary MR angiography with real-time navigator correction: comparison of navigator locations. Radiology 212, 579-587 (1999).

17. Mavrogeni, S., et al. Magnetic resonance angiography is equivalent to X-ray coronary angiography for the evaluation of coronary arteries in Kawasaki disease. J. Am. Coll. Cardiol. 43, 649-652 (2004).

18. Zhu, C., et al. Isotropic 3D black blood MRI of abdominal aortic aneurysm wall and intraluminal thrombus. Magn. Reson. Imaging 34, 18-25 (2016).

19. Eikendal, A. L., et al. 3D black blood VISTA vessel wall cardiovascular magnetic resonance of the thoracic aorta wall in young, healthy adults: reproducibility and implications for efficacy trial sample sizes: a cross-sectional study. J. Cardiovasc. Magn. Reson. 18, 20 (2016).

20. Jara, H., Yu, B. C., Caruthers, S. D., Melhem, E. R. \& Yucel, E. K. Voxel sensitivity function description of flow-induced signal loss in MR imaging: implications for black-blood MR angiography with turbo spin-echo sequences. Magn. Reson. Med. 41, 575-590 (1999).

21. Qiao, Y., et al. Intracranial arterial wall imaging using three-dimensional high isotropic resolution black blood MRI at 3.0 Tesla. J. Magn. Reson. Imaging 34, 22-30 (2011).

22. Fan, Z., et al. Carotid arterial wall MRI at 3T using 3D variable-flip-angle turbo spin-echo (TSE) with flow-sensitive dephasing (FSD). J. Magn. Reson. Imaging 31, 645-654 (2010).

23. Mihai, G., Chung, Y. C., Merchant, A., Simonetti, O. P. \& Rajagopalan, S. T1-weighted-SPACE dark blood whole body magnetic resonance angiography (DB-WBMRA): initial experience.

J. Magn. Reson. Imaging 31, 502-509 (2010).

24. Onouchi, Z., Shimazu, S., Kiyosawa, N., Takamatsu, T. \& Hamaoka, K. Aneurysms of the coronary arteries in Kawasaki disease. An angiographic study of 30 cases. Circulation 66, 6-13 (1982).

25. Makowski, M. R., et al. Characterization of coronary atherosclerosis by magnetic resonance imaging. Circulation 128, 1244-1255 (2013).

26. Ozolanta, I., Tetere, G., Purinya, B. \& Kasyanov, V. Changes in the mechanical properties, biochemical contents and wall structure of the human coronary arteries with age and sex. Med. Eng. Phys. 
20, 523-533 (1998).

27. Schär, M. et al. The impact of spatial resolution and respiratory motion on MR imaging of atherosclerotic plaque. J. Magn. Reson. Imaging 17, 538-544 (2003).

28. Fayad, Z. A., et al. Noninvasive in vivo human coronary artery lumen and wall imaging using blackblood magnetic resonance imaging. Circulation 102, 506-510 (2000).

29. Botnar, R. M. et al. Noninvasive coronary vessel wall and plaque imaging with magnetic resonance imaging. Circulation 102, 2582-2587 (2000).

30. Suzuki, A., et al. Functional behavior and morphology of the coronary artery wall in patients with Kawasaki disease assessed by intravascular ultrasound. J. Am. Coll. Cardiol. 27, 291-296 (1996).

\section{Figures}

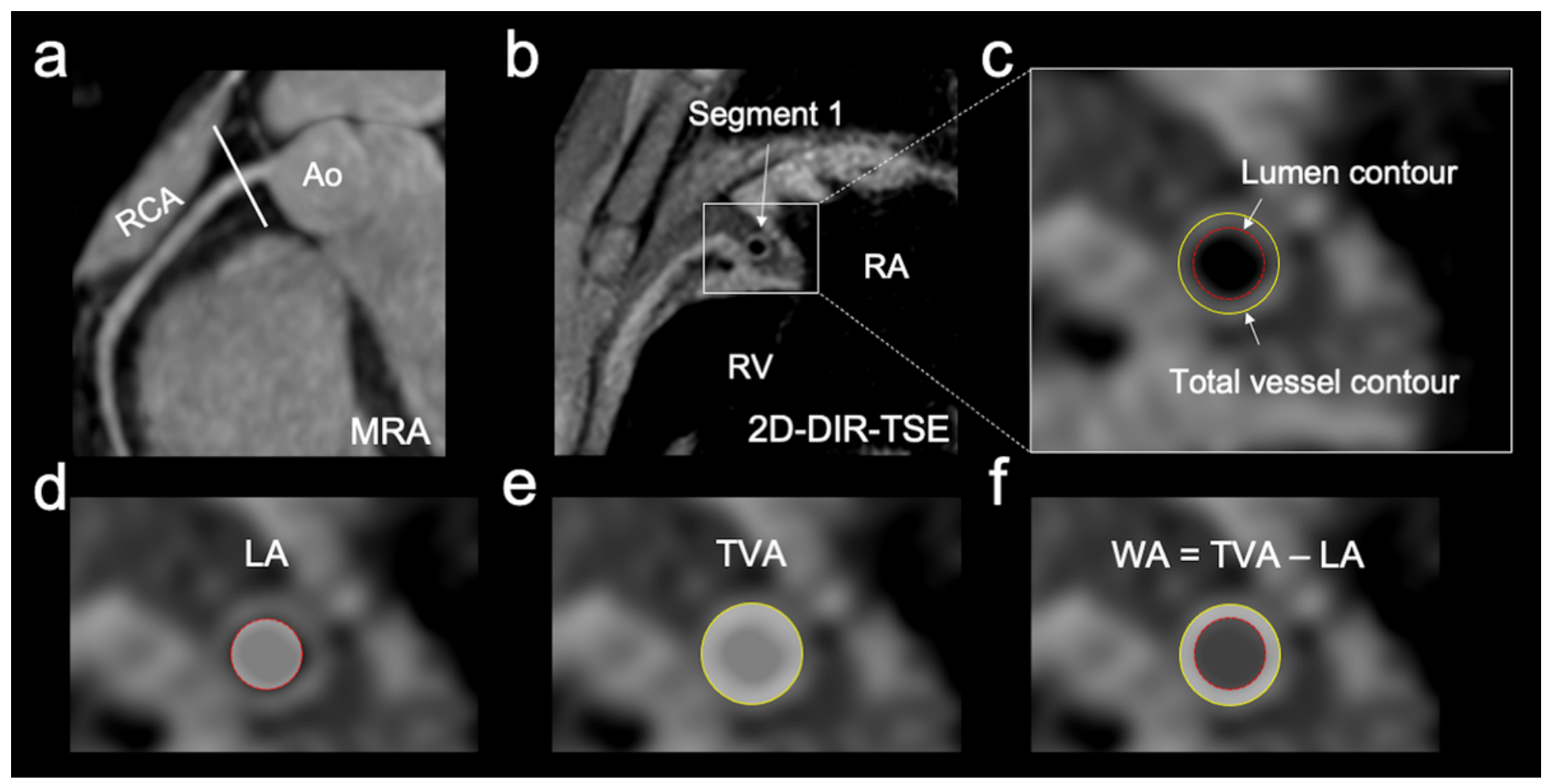

Figure 1

Regions of interest segmentation. The coronary magnetic resonance angiography-collapsed partial maximum intensity projection image demonstrates right coronary artery (RCA) segments 1 in a 19-yearold boy with Kawasaki disease (a). At 6 months of age, he experienced a coronary artery aneurysm of up to $5.9 \mathrm{~mm}$ in RCA segments 1 . It is currently involuted and divided into normal regions. The white line depicts the slice position in the two-dimensional dual inversion-recovery turbo spin-echo cross-sectional image $b$. Image $c$ is an enlarged image of the measurement location of $b$. The lumen of the coronary artery (red dotted line) and the boundaries of the wall (yellow solid line) have been manually contoured (c). Subsequently, the lumen area (LA), the total vessel area (TVA) and the wall area (WA) have been measured ( $d, e, f)$ (Ao, aorta; RA, right atrium; and RV, right ventricle). 


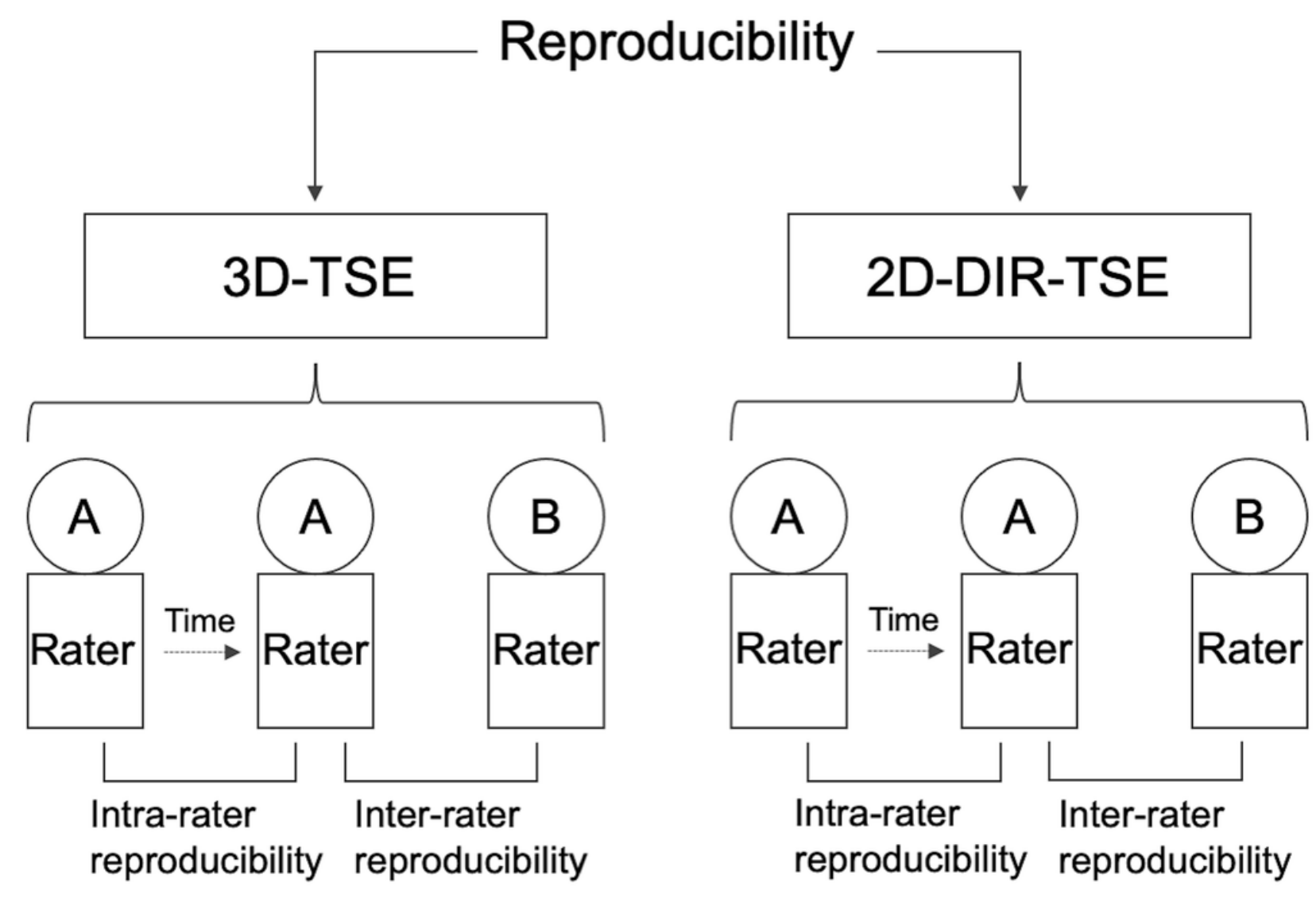

Figure 2

A graphic overview of inter- and intra-rater image analysis to determine reproducibility of 3D-TSE and 2DDIR-TSE images. 


\section{Aneurysmal regions}
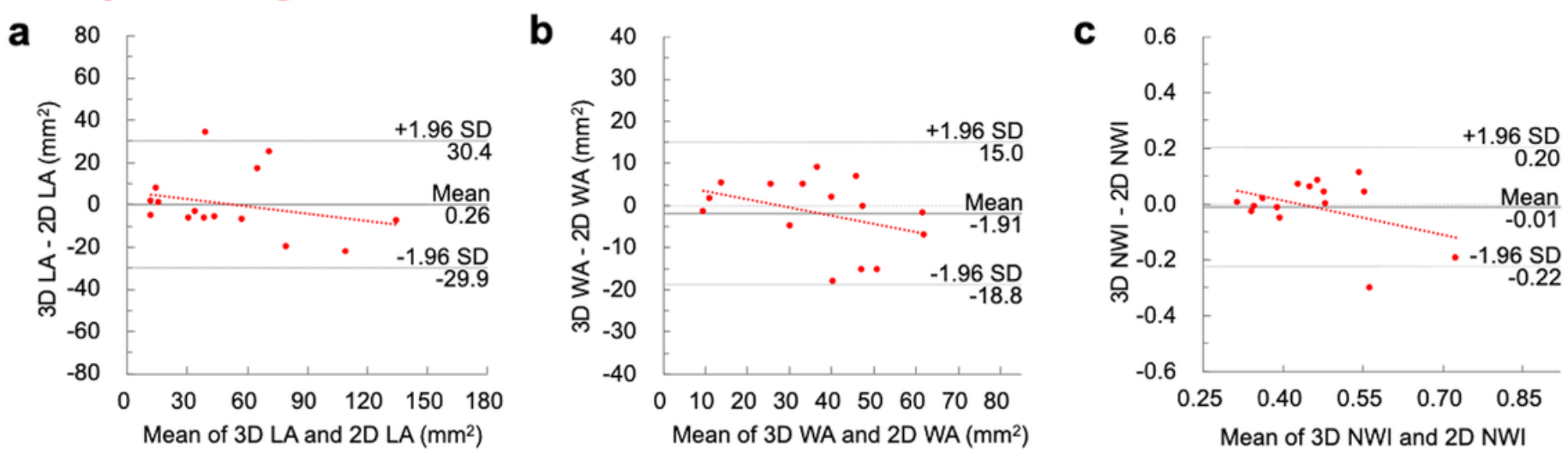

\section{Normal regions}
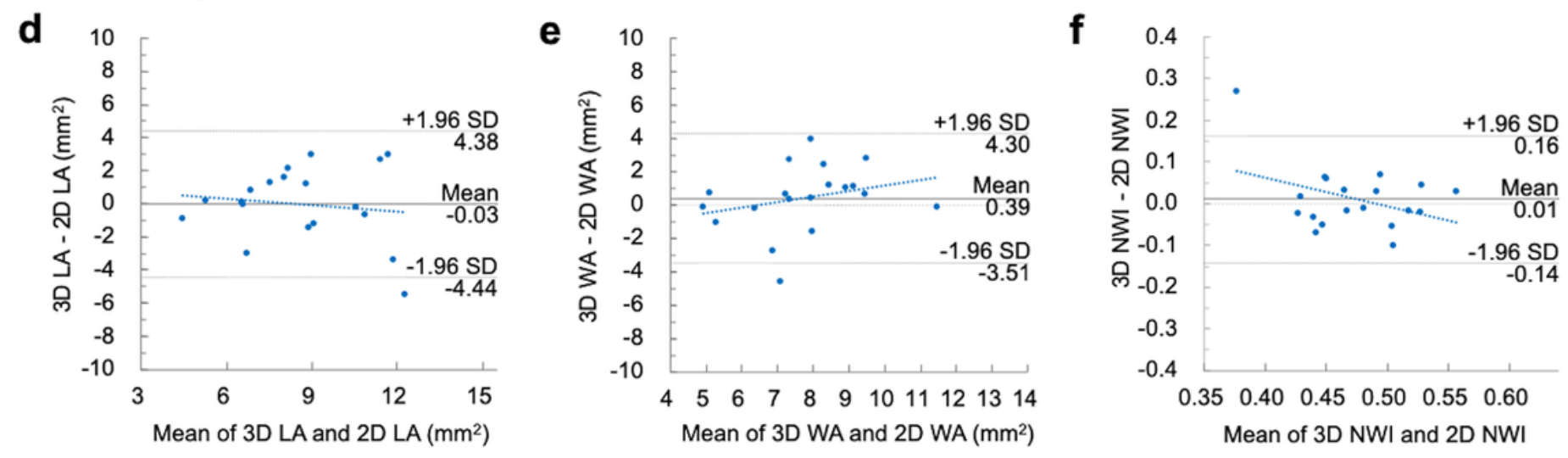

\section{Figure 3}

Bland-Altman plots of the lumen area (LA), wall area (WA), and normalized wall index (NWI), determined by three-dimensional turbo spin-echo (3D-TSE) and two-dimensional dual inversion recovery turbo spinecho (2D-DIR-TSE) images in aneurysmal $(a, b, c)$ and normal $(d, e, f)$ regions. Bland-Altman plots demonstrate good agreement between 3D-TSE and 2D-DIR-TSE images. In the aneurysmal region, there are no proportional biases in LA, WA, and NWI ( $P=0.334,0.167$, and 0.136 , respectively). Similarly, in the normal regions, there are no proportional biases in $L A, W A$, and NWI $(P=0.600,0.259$, and 0.105 , respectively). 


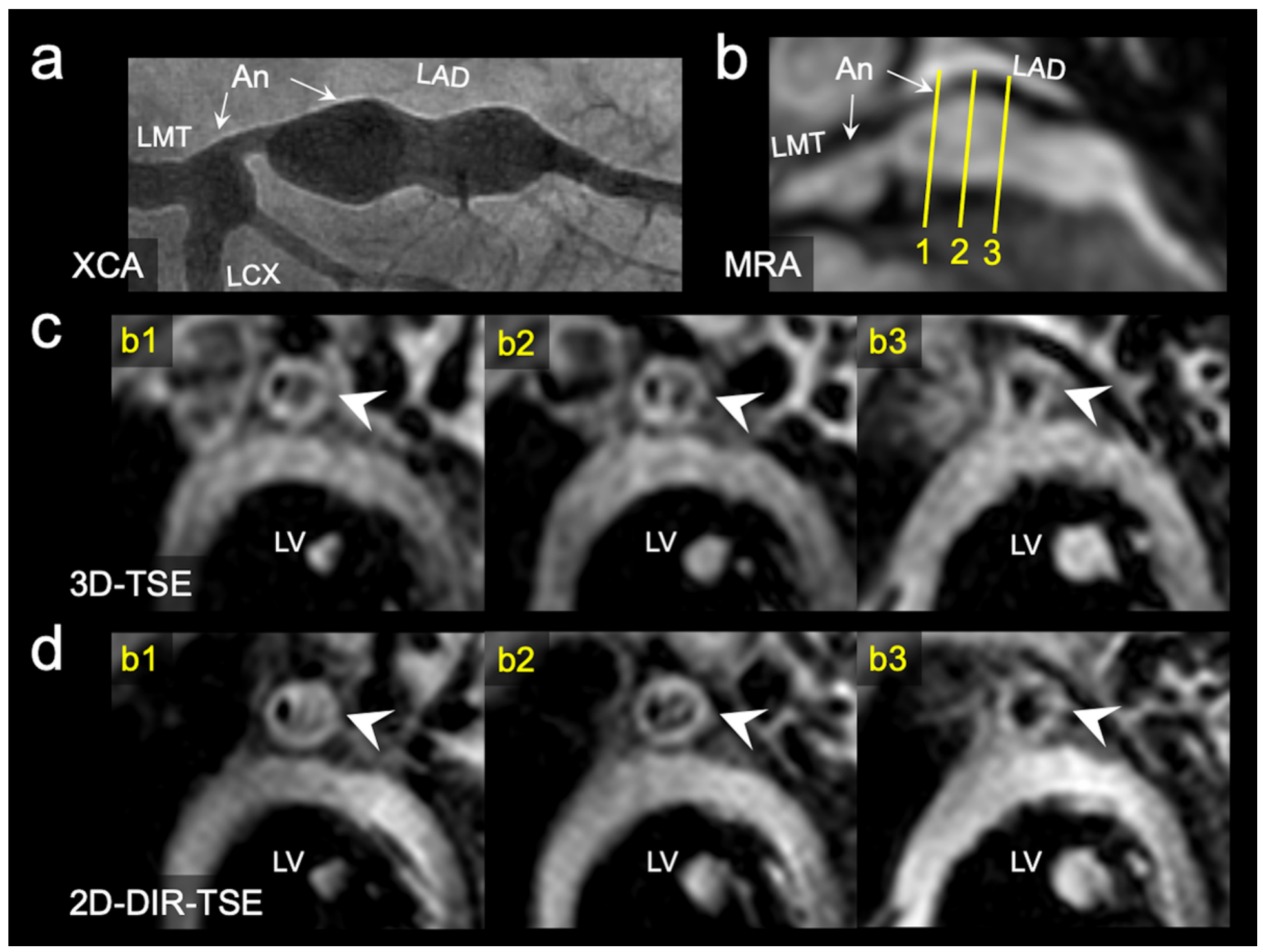

Figure 4

X-ray coronary angiography images demonstrate two left coronary artery aneurysms (coronary artery segment 5, $4.5 \mathrm{~mm}$; segment 6 to 7, $8.3 \mathrm{~mm}$ ) in an 8-year-old boy with Kawasaki disease (a). In the magnetic resonance angiography-collapsed partial maximum intensity projection image, the yellow lines indicate aneurysmal regions (b). Each cross-sectional image of three-dimensional turbo spin-echo (3DTSE) and two-dimensional dual inversion recovery turbo spin-echo (2D-DIR-TSE) corresponds to the solid line numbers in the MRA (c and d). In the 3D-TSE and 2D-DIR-TSE images, intraluminal signals in the aneurysm mimics thrombi because the flow signals are not suppressed. In the cross-section indicated by b2, the visual grading of the lumen boundary and outer wall boundary in 3D-TSE and 2D-DIR-TSE images have been grade 2 and 4, respectively, and depicted equally (arrowheads in c and d). (An, aneurysm; LMT, left main trunk; LAD, left anterior descending artery; LCX, left circumflex artery; and LV, left ventricle). 